\title{
FLUCTUATION OF CONTACT VOLTAGE DURING FRETTING PHASES
}

\author{
Rochdi El Abdi', Sofiane El Mossouess ${ }^{2}$, Noureddine Benjemâa ${ }^{3}$, \\ ErwannCarvou 4 , Laurent Doublet ${ }^{5}$ \\ ${ }^{1}$ Université de Rennes1- Institut de Physique de Rennes- M\&V \\ Campus de Beaulieu, 35042 Rennes Cedex France \\ 2 Université de Rennes1- Institut de Physique de Rennes \\ Campus de Beaulieu, 35042 Rennes Cedex France \\ ${ }^{3}$ EntrepriseContelec- 8, Rue de la Motte Baril, 35000 Rennes-France \\ 4 Université de Rennes1-Institut de Physique de Rennes \\ Campus de Beaulieu, 35042 Rennes Cedex France \\ ${ }^{5}$ EntrepriseValéo, Etudes Electroniques-Créteil, Europarc. 94000 Créteil -France \\ relabdi@univ-rennes1.fr
}

\begin{abstract}
Due to repetitive micro-displacements, the fretting phenomenon was defined as an electrical and mechanical degradation of the electrical contact interface in telecommunications and more recently in power automotive connectors. Commonly, the electrical degradation was quantified by the increase of contact resistance deduced from the contact voltage. This work aims to address the analysis of the change of contact temperature and the contact voltage during the three successive fretting phases at low and high level contacts. U-shape samples of cooper with a tin protective coating were used and fixed on a vibration system.

Indeed, the analysis of the voltage fluctuations in term of level and time appearance was a very useful tool to analyze in-situ the fretting behaviour. Upon the analysis of experimental data, it was found that the first phase corresponds to the phase of sample self-cleaning and to the beginning of the fretting phenomenon; the second phase corresponds to a rapid increasing of the fretting phenomenon. Finally, at the end of the second phase, the fretting phenomenon is stable and the measured contact temperature reached $150{ }^{\circ} \mathrm{C}$.
\end{abstract}

Keywords: Electrical Contact, Fretting Phenomenon, Contact Resistance, Contact Zone, Vibration Cycles.

\section{Introduction}

In automotive and aerospace industry, power connection is constantly increasing.

Therefore, the on-board electrical power and the number of power connections such as printed circuit boards (PCB) increase significantly and the power connector was to be one of the major electric system used in the power connections. However, this development can't be at the expense of system reliability for operational and economic reasons. It is indeed necessary that electric systems offer a reliability level which must be equivalent or even superior to the current systems. The automotive environment was particularly harsh for the electronic components and the electrical wiring systems. There are many constraints (temperature, vibration, humidity ...) which are not compatible with the use of electrical components.

To ensure a good performance of the electrical connections in harsh environments, connector manufacturers have used plating contacts with noble materials such as gold or silver. Indeed, the noble materials can fight against the corrosion while ensuring a good electrical resistance.

However, due to the cost more and higher, manufacturers of electronic components have chosen to reduce the gold thickness and the plating surface. The decrease of the material thickness led to a fast wear of contact surfaces inside electrical components which leads to the voltage increase due to the fretting corrosion phenomenon [1].

In this work, the behaviour of an electrical contact surface was studied when subjected the several cycles' vibration and the change of the contact surface wear was analyzed.

\subsection{Samples Used and Experimental Set-Up}

The present study analyzed the contact voltage change for copper alloy samples of $0.6 \mathrm{~mm}$ in thickness with a tin coating of $1 \mu \mathrm{m}$ in thickness. 
The samples were produced with a sheet of 20 $\mathrm{mm}$ width, using the processing technique of stamping and bending.

The instrumented device consisted in applying indentation and insertion forces on two U-shaped samples (Fig. 1). One of the two samples was flat and the other contained a sphere with a radius $\mathrm{R}$ equal to $3 \mathrm{~mm}$ (Fig. 1). The two samples were fixed on the Teflon part (Fig. 2). Before each experiment, the sample surface was wiped by an antioxidant paste and then the sample was cleaned in an ultrasonic alcohol bath.

The experimental set-up consists of a shaker (LDS V555 with a maximum force of 939N, maximum acceleration of $100 \mathrm{~g}\left(\mathrm{~g}=9.81 \mathrm{~m} / \mathrm{s}^{2}\right)$ ) which can apply sinusoidal vibrations with an amplitude greater than $1 \mu \mathrm{m}$ and a frequency between $50 \mathrm{~Hz}$ and $100 \mathrm{~Hz}$. A sinusoidal vibration was applied on the lower part of the horizontal linear stage (Fig. 2) which was attached to a slippery table (horizontal displacement between $(-a)$ and $(+a))$.

The upper part of the sample was attached to a vertical slippery part. A contact force of $3 \mathrm{~N}(\mathrm{Fc})$ was applied with the help of a mass.
Two displacement sensors using laser light $(0.01 \mu \mathrm{m}$ of resolution) were used to measure the horizontal displacement of the lower sample and to give the vertical displacement of the upper sample.

A D.C. current generator was installed in order to transmit an electrical current of $10 \mathrm{~A}$ with the help of a power supply of $14 \mathrm{~V}$. The electrical measurements of the contact voltage were obtained with two oscilloscopes (Tektronix DP05000 and TDS5000); one was used to give the contact voltage measurement and the second to detect the electrical arc appearance.

In addition a thermo-couple was used to measure the temperature near the contact zone and to stop the current source if the temperature goes beyond $150^{\circ} \mathrm{C}$.

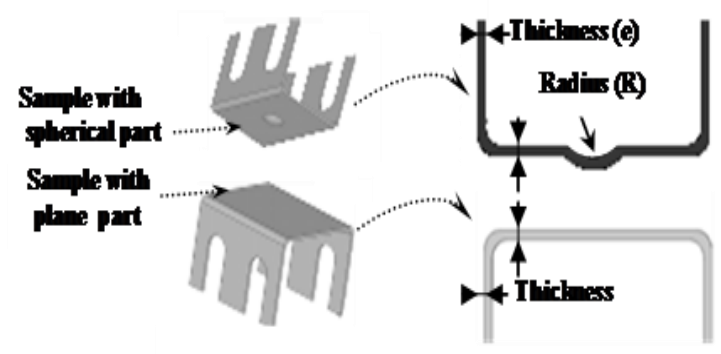

Figure 1: Samples used

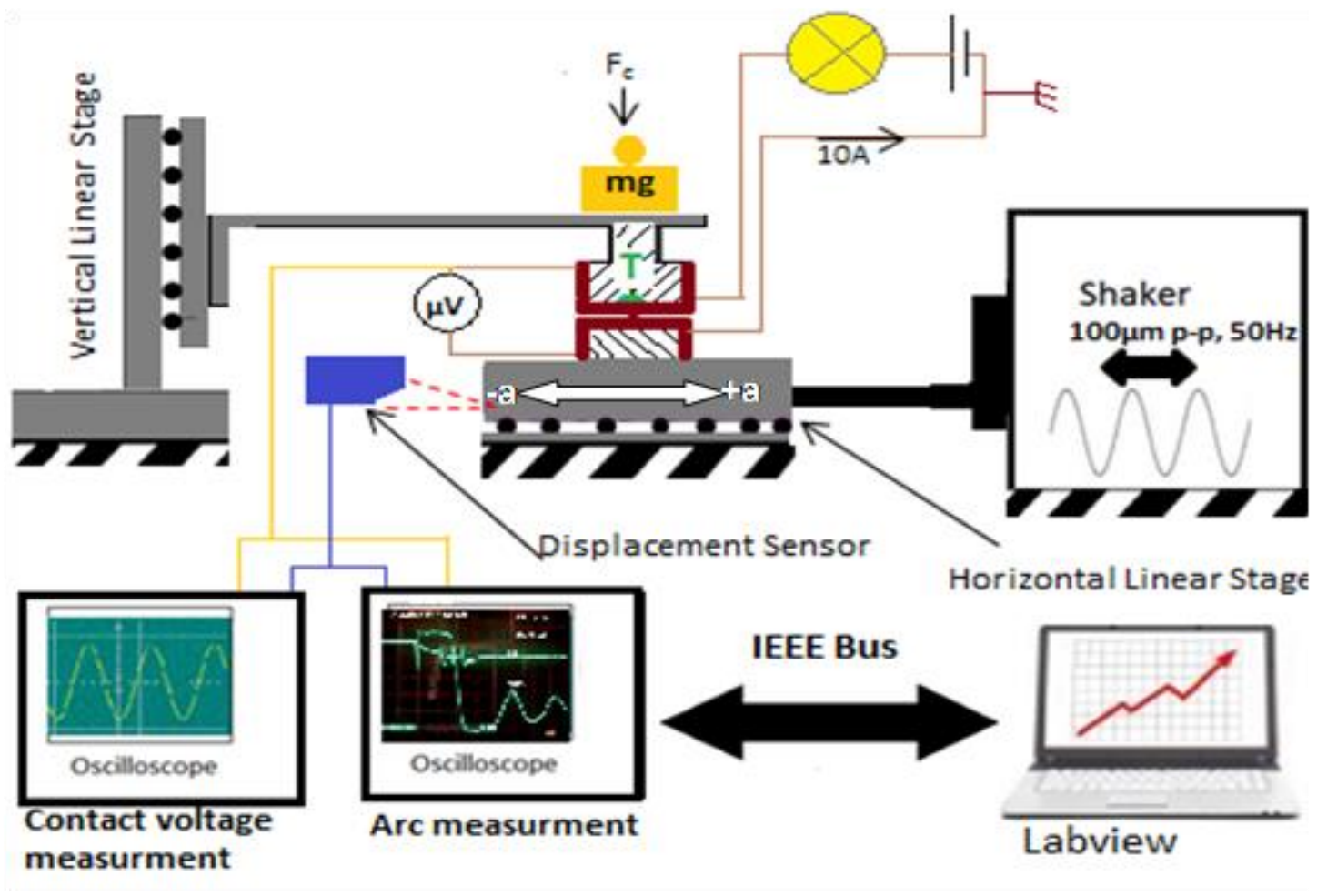

Figure 2: Experimental set-up used

\section{Study of Contact Voltage during the Three Fretting Phases}

Two "U" samples (plane part and spherical part) were submitted to the shaker vibrations and are moving at frequency $50 \mathrm{~Hz}$ with amplitude of $2 \mathrm{a}=$
$0.8 \mathrm{~mm}$ (peak to peak, Fig. 2) of sinus oscillations. This movement is controlled and measured by a displacement sensor (using laser with $0.1 \mu \mathrm{m}$ of resolution) focused.

These parameters simulate real conditions of use of a contact in the automotive connectors [2-5]. 
Figure 3 gives the change of contact voltage and contact temperature measured near the contact interface between the two sample parts versus the number of vibration cycles.

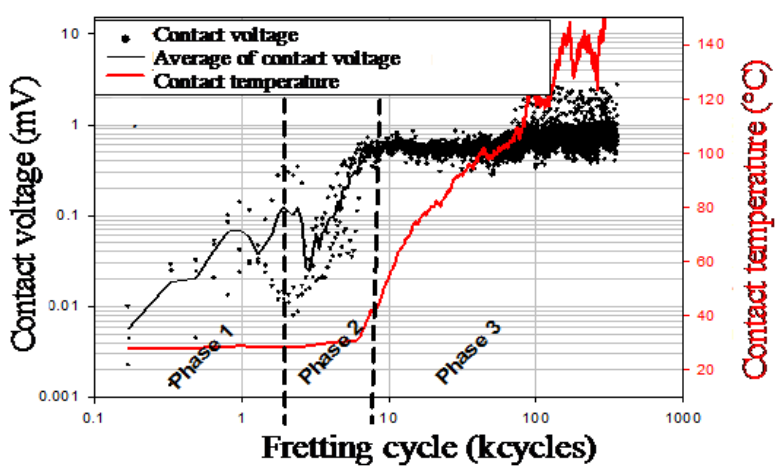

Figure 3: Contact voltage and contact temperature versus cycle number (frequency $=50 \mathrm{~Hz}$, total displacement amplitude $2 a=50 \mu \mathrm{m}$, current $\mathrm{I}=10 \mathrm{~A}$ )

Three vibration phases were obtained. The first phase (from 0 kcycles to 2.5 kcycles), corresponds to the self-cleaning contact track and was the beginning of the fretting phenomenon. The second phase (from 2.5 kcycles to 8 kcycles) corresponds to the acceleration of the fretting phenomenon and stops at the saturation voltage. Finally, after 8 kcycles, there has been little change in the fretting phenomenon and the vibration test stops when the measured contact temperature reached $150{ }^{\circ} \mathrm{C}$.

The change in temperature follows the one of the contact voltage, namely during the first phase, but at the end of the second vibration phase, a rapid temperature increase was obtained.

The temperature increase was due to fluctuating potentials whose origins were due to heating caused by electric arcs $\left(6000^{\circ} \mathrm{C}\right)$ and Joule's effect dissipated in the contact interface [6].

However, the heating goes on during the third phase, despite the stabilization of the contact voltage. This phenomenon is due to the inertia and heat dissipation.

A comparison between the changes of contact voltages and phase cycle number was made for a low $(\mathrm{I}=200 \mathrm{~mA}, \mathrm{~V}=5 \mathrm{~V})$ and a high $(\mathrm{I}=10 \mathrm{~A}, \mathrm{~V}=16 \mathrm{~V})$ levels fretting contact (Figs. 4, 5).

The duration of phases 1 and 2 for the low contact level was lower than the one obtained during the high level contact (Fig. 4). But the durations of the third phase were close in both level conditions.

During the phases 1 and 2, the electrical potential increase follows the current in-crease, especially during the first phase (Fig. 5). Indeed, during the beginning of fret-ting in the first phase, the electrical conduction occurs essentially between metallic spots and remains linear. As for the third phase, the current increase does not generate additional potential (the contact voltages for the two levels were equal) and the contact voltage reaches the breakdown voltage.

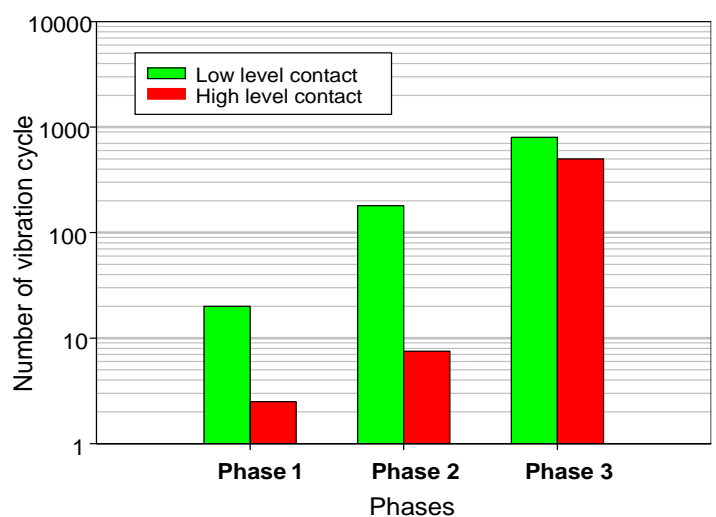

Figure 4: Cycle number for three phases for low ( $I=200$ $m A, V=5 \mathrm{~V})$ and high $(I=10 \mathrm{~A}, \mathrm{~V}=16 \mathrm{~V})$ levels fretting contact

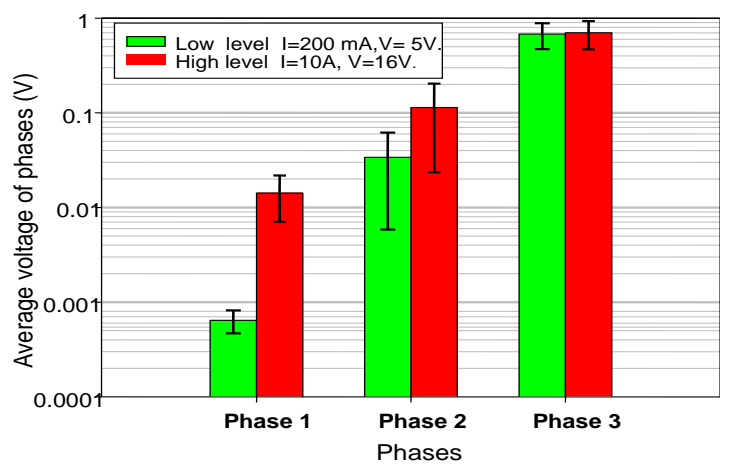

Figure 5: Cycle number for three phases for low ( $I=200$ $m A)$ and high ( $I=10 A)$ levels fretting contact

Figure 6 shows for different cycle numbers, the change of the contact voltage for a high level contact. Two contact voltage values were observed: a minimum value Vs obtained for 0 cycles (contact voltage without vibrations) and a maximum value $V_{d}$ characterized by voltage peaks.

The voltage peaks move to both ends of the contact track (see voltage contact change after 0.75 kcycles). This was related to the constriction of the current lines in granular media.

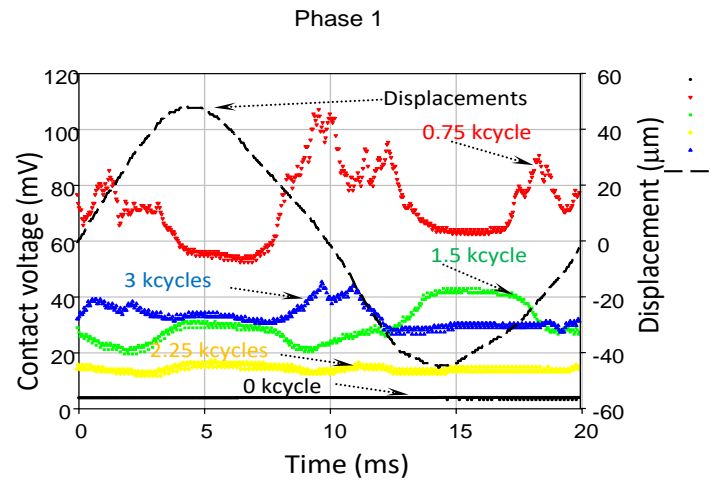

Figure 6: Change of contact voltage (high level contact) during the first fretting phase $1(10 \mathrm{~A}, 50 \mathrm{~Hz}$ $2 a=50 \mu \mathrm{m}$ ) 
Figure 7 gives the voltage changes along the contact track in both vibration directions (Right way and Left way), during the phase 1 , when the plane part of the "U" sample moves alternately from right to left direction (Fig. 2).

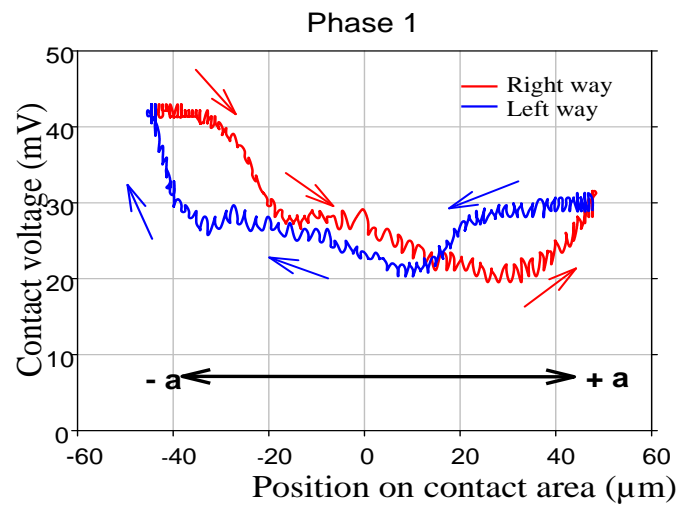

Figure 7: Contact voltage versus displacement of contact area during the first fretting phase 1

The change analysis of the contact voltage shows that the tension peaks appear in the two directions of vibration (positive direction and negative direction) at both ends of the contact track with different values $(\mathrm{V} \approx 43 \mathrm{mV}$ for the end of the left contact track and $V \approx 32 \mathrm{mV}$ for the end of the right contact track) (Fig. 7).

On the left half of the contact track, the contact voltages were more important when the plane part of "U" sample goes towards the positive direction (Right way, Fig. 7), while on the right half, the contact voltages were greater when the plane part of " $U$ " sample goes towards the negative direction (left way).

During the second phase of vibration, the contact voltage behavior remains unchanged and the same as in the first phase (Fig. 8).

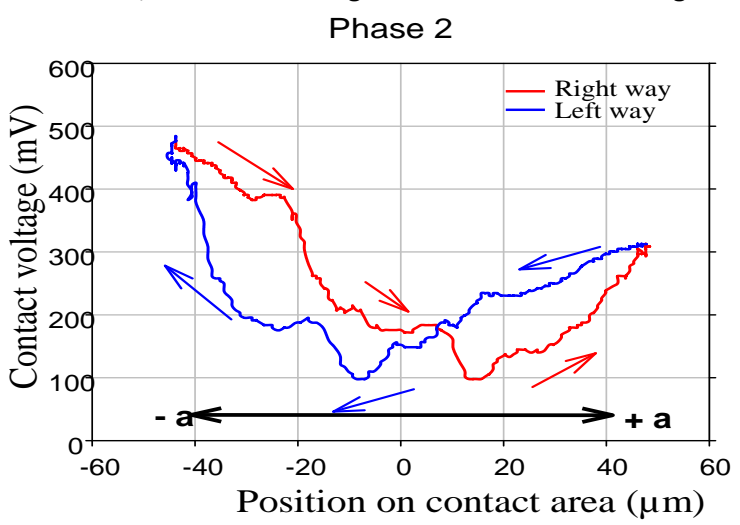

Figure 1: Contact voltage versus displacement of contact area during the second fretting phase 2

Along the contact track during the third vibration phase, the contact voltage becomes unpredictable (Fig. 9) and depends on the resistive film behavior which was deposited on the contact surface.

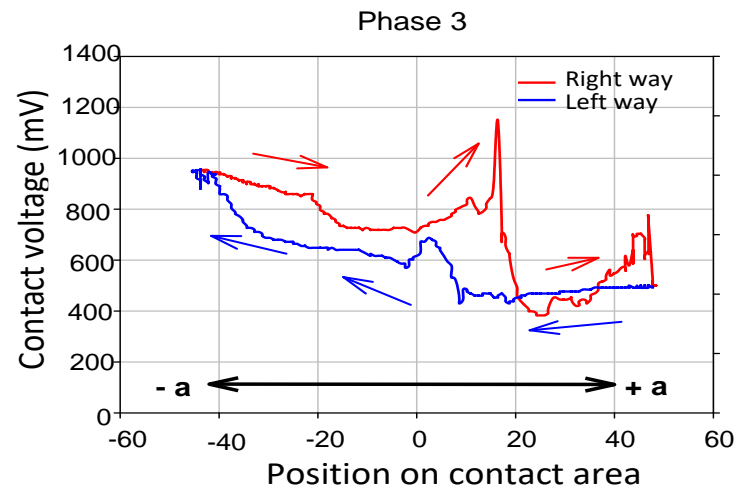

Figure 2: Contact voltage versus displacement of contact area during the third fretting phase 3 


\section{Study of Contact Area Worn out by Friction during the Three Fretting Phases}

Figure 10 gives the wear contact zones (with X100 magnification) after a vibration test with a frequency of $100 \mathrm{~Hz}$, a current of $10 \mathrm{~A}$ and a shaker displacement of $50 \mu \mathrm{m}$.
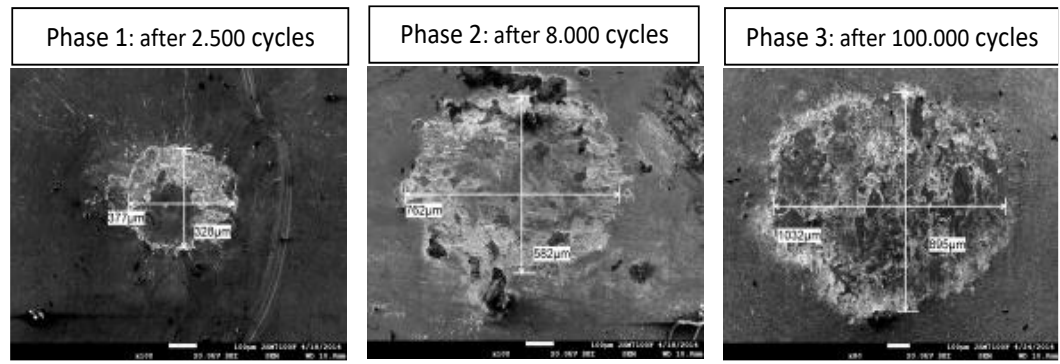

Figure 10: Size of contact zones at the end of the three phases of fretting

After these tests, a chemical analysis using the Energy-dispersive X-ray spectroscopy (EDX) was undertaken to quantify the different chemical
The tests were stopped at different stages of fretting. At the end of the first phase, the size of the contact track reached $377 \mu \mathrm{m}$ and reached $762 \mu \mathrm{m}$ and $1032 \mu \mathrm{m}$ at the end of the second and third phase respectively.

The track sizes have nearly tripled between the first and the third phase of vibration (i.e. between 2 kcycles and 100 kcycles).
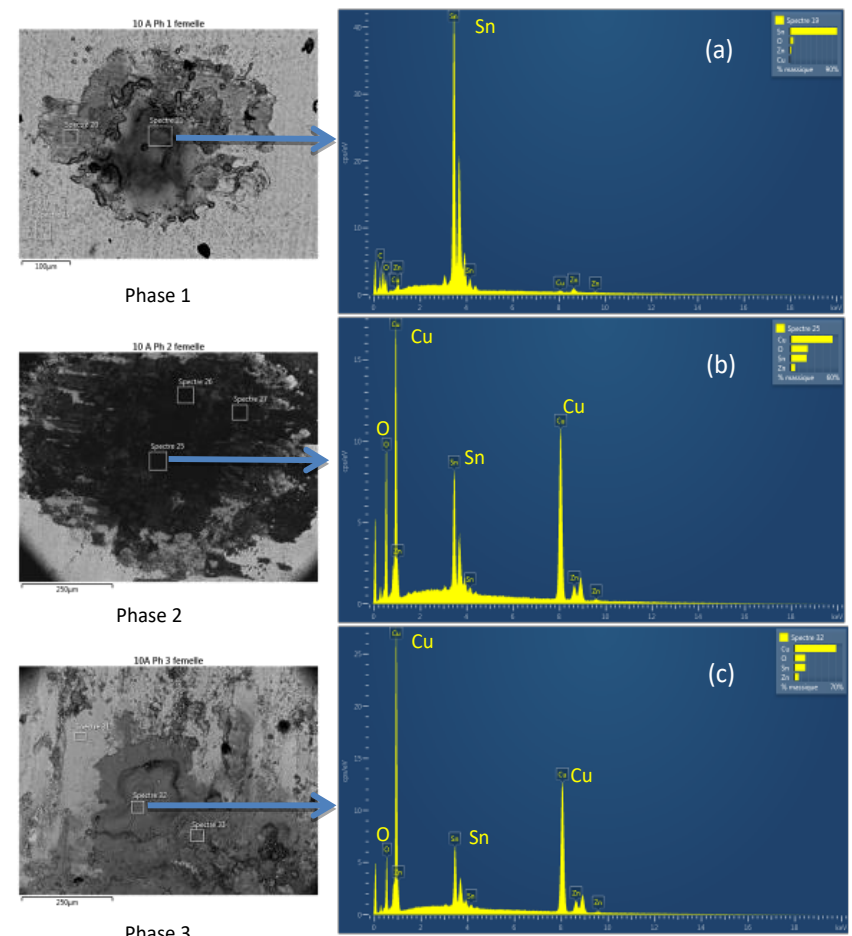

Figure 11: Energy-dispersive X-ray spectroscopy of track at the end of each fretting phase

During the first vibration phase, a high tin proportion ( $\mathrm{Sn} \approx 43 \%$ ) was detected. Other chemical elements with small proportions were found $\mathrm{CO} \approx$ $4 \%$ and $\mathrm{Cu} \approx 2 \%$ ) (Fig. $11 \mathrm{a}$ ). An increase of the substrate percentages ( $\mathrm{Cu}$ ) and oxygen (O) during the second phase was obtained $(0 \approx 10 \%, \mathrm{Cu} \approx 9 \%)$ (Fig.11b). During the last phase, the copper proportion increases $(\mathrm{Cu} \approx 27 \%$ ) (Fig.11c), while the percentage of the coating de-creases considerably $(\approx$ element percentages on the damaged contact surface at different vibration phases (Fig. 11).
$9 \%$ during the second phase and $\approx 6 \%$ during the third phase).

Curves of the atomic percentages for each chemical element were obtained (Fig. 12).

The different chemical elements come from the substrate $(\mathrm{Cu}$ and $\mathrm{Zn})$, from the coating $(\mathrm{Sn})$ and Oxygen (0).

The coating was gradually removed during the first and second vibration phases and disappeared in 
the third phase where the contact voltage was high. In addition, during the third phase, the oxide debris mainly consist on copper and oxygen.

Finally, the oxygen concentration increases during the first phase and remains high during the second and third phase. We assume that the oxygen and tin were bound to give $\mathrm{SnO} 2$ during the initial phase. But in the third phase, oxygen was bound to cop-per forming $\mathrm{Cu} 2 \mathrm{O}$.

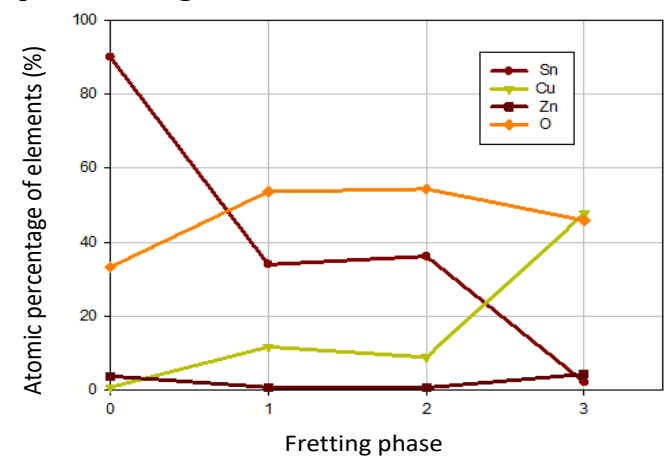

Figure 12: Atomic percentage of elements on contact surface during the three phases of fretting

\section{Conclusion}

A high level current accelerates the appearance of the first and second fretting phases and increases the temperature value at the contact interface due to Joule's effect.

The change analysis of the contact voltage shows that the tension peaks appear in the two directions of vibration (positive and negative directions) at both ends of the contact track with different values.

On the other hand, the contact voltages on the left half and right half of the contact track are different and depend on the direction displacement.

The size of the contact zone worn out by friction has tripled between the beginning of the vibration test (phase1) and the final vibration cycles (phase 3).
EDX analysis of the contact zones has shown that different chemical elements appear at different vibration phases. Indeed, during the first damage of the contact surface which corresponds to the fretting phase 1 , there were little of elements other than tin on the contact surface. The increased wear during the second phase led to the appearance of the copper layer and oxide debris (presence of oxygen), while the percentage of tin drops significantly during the third stage.

\section{References}

[1] Benjemâa, N.,Carvou,E.: Electrical Contact Behavior of Power Connector during Fretting Vibration. Proc. of 52nd IEEE Holm Conference on Electrical Contacts, Montréal. 2006; 263.

[2] Heaton,C.E., McCarthy, S. L.:High Cycle Fretting Corrosion Studies on Tin-Coated Contact Materials. Proceedings of the Forty-Seventh IEEE Holm Conference on Electrical Contacts (IEEE Cat. No.01CH37192). 2001; 209.

[3] Maul,C., Mc Bride,J. W., Swingler, J.:Intermittency Phenomena in Electrical Connectors. IEEE Trans. Components Packag. Technol. 2001; 24, 3, 370.

[4] Malucci,R.D.: Impact of Fretting Parameters on Contact Degradation. In Electrical Contacts 1996. Proceedings of the Forty-Second IEEE Holm Conference on Electrical Contacts joint with the 18th International Conference on Electrical Contacts, 1996; 395.

[5] Timsit,S.: Electrical Contact Resistance: Properties of Stationary Interfaces. Electrical Contacts. Proceedings of the Forty-Fourth IEEE Holm Conference on Electrical Contacts (Cat. No.98CB36238), 1998; 1.

[6] Holm,R.: Electrical Contacts: Theory and Applications, Fourth Edition. Springer-Verlag. Hardcover. Berlin, 1999. 